\title{
LES TRAUMATISMES EXTERNES DU LARYNX
}

\author{
S. TRABELSI, A. HACHICHA, N. BELTAIEF, A. CHARFEDDINE , S.TABABI, \\ R. ZAININE , E. MNIF* ${ }^{\star}$ S. SAHTOUT , G. BESBES. \\ SERVICE ORL ET CHIRURGIE CERVICOFACIALE. \\ *SERVICE D'IMAGERIE MÉDICALE. \\ HÔPITAL LA RABTA -TUNIS
}

\begin{abstract}
RESUME
Objectif : Les traumatismes externes du larynx sont rares. Ces traumatismes, ouverts ou fermés, peuvent être isolés, ou s'intégrer dans la cadre d'un traumatisme cervical complexe ou d'un polytraumatisme. Leurs aspects étiologiques, cliniques, diagnostiques et thérapeutiques sont très variés. Les principes généraux de leur prise en charge et de leur traitement chirurgical dépendent de la gravité des lésions

Matériel et méthodes : Nous rapportons une étude rétrospective portant sur 30 patients ayant été suivis et traités pour un traumatisme externe du larynx dans le service d'ORL de la Rabta. La série a été colligée sur une période de 25 ans allant de 1983 à 2008.

Résultats : L'âge moyen de nos patients est de 30 ans. Les étiologies sont dominées par les accidents de la circulation (53\% de nos patients). Tous nos malades ont eu un examen endolaryngé par le nasofibroscope ou le miroir laryngé. La laryngoscopie sous AG a été réalisée chez 25 patients. La tomodensitométrie cervicale a été pratiquée chez 16 patients. Au décours de ces examens, tous nos patients ont été classés selon la classification de Schaeffer modifiée par Fuhrman. Plus que la moitié des patients (54\%) sont classés en stade I et II. L'exploration chirurgicale a été indiquée pour 16 patients. Le traitement a été conservateur dans tous les cas. Le recul moyen est de 2 ans. Trois de nos patients ont développé une sténose sous glottique. Deux d'entre eux ont eu une laryngoplastie d'agrandissement type Réthi. Conclusion : Tout traumatisme laryngé découvert, doit être pris en charge correctement afin d'éviter des complications pouvant mettre en jeu aussi bien le pronostic vital que le pronostic fonctionnel.
\end{abstract}

Mots-clés : nasofibroscopie laryngée, tomodensitométrie cervicale, sténose laryngée, rééducation orthophonique

Objective : Laryngeal injuries are rare. They can be isolated or associated with fractures of the facial skeleton. Early diagnosis and proper initial management may sometimes be difficult or delayed.

The indication for surgery depends on the extent of the injury.

Materials and methods : We report a retrospective review including thirty patients with laryngeal trauma treated over a 25-year period

Results : The mean age was 30 years. Commonest cause of injury was cut throat injuries followed by road traffic accidents. Fibreoptic endoscopy was performed for all the patients. Ct scan was done for 16 patients. All the patients were classified selon la classification de Schaeffer.

More then the half (54\%) was classified in stage I and II. Surgery was indicated for 16 patients. The treatment was conservative for all the cases. The middle recurrence was 2 years. Three of our patients developped a sous glottic stenosis. Two of them had a laryngoplasty type Rethi.

Conclusion : Their symptoms are variable ranging from obvious airway obstruction to minor or almost missing symptoms. A coordinated team approach is necessary for proper management of these injuries.

Keywords : Fibreoptic endoscopy, cervical CT-Scan, laryngeal stenosis, voice therapy sessions

\section{INTRODUCTION}

Les traumatismes externes du larynx sont rares. Ces traumatismes, ouverts ou fermés, peuvent être isolées, ou s'intégrer dans un traumatisme cervical complexe ou dans le cadre d'un polytraumatisme. Leurs aspects étiologiques, cliniques, diagnostiques et thérapeutiques sont très variés. Les principes généraux de leur prise en charge et de leur traitement chirurgical dépendent de la gravité des lésions.

\section{MATÉRIEL ET MÉTHODES}

II s'agit d'une étude rétrospective portant sur 30 patients porteurs d'un traumatisme externe du larynx colligés sur une période de 25 ans, allant de 1983 à 2008.

Nous avons étudié l'âge, le sexe, les antécédents pathologiques ainsi que les signes d'appel amenant les patients à consulter.

L'examen laryngé au nasofibroscope ou au miroir laryngé a été réalisé chez tous les patients. La tomodensitométrie 
cervicale a été pratiquée pour 16 patients.

Quand un traitement chirurgical a été indiqué, la voie d'abord était cervicale.

L'évolution post opératoire, a été jugée sur des critères cliniques et endoscopiques.

\section{RESULTATS}

L'âge moyen de nos patients était de 30 ans avec des extrêmes allant de 4 à 55 ans. Un pic de fréquence a été constaté au niveau de la 3e décade. Le sex-ratio était de 9 avec une nette prédominance masculine.

Les étiologies étaient dominées par les accidents de la circulation (53\%). Les agressions étaient retrouvées dans $27 \%$ des cas, suivies des accidents de travail et des autolyses (12\%).

Ces traumatismes s'intégraient dans le contexte d'un polytraumatisme dans $27 \%$ des cas. Ils étaient fermées dans 19 cas (64\%) et ouverts dans 11 cas (36\%).

La dysphonie était le symptôme fonctionnel le plus commun (84\%). 57\% des patients se sont présentés à nos urgences avec une dyspnée. La dysphagie a été retrouvée dans $27 \%$ des cas. D'autres signes ont été constatés tels qu'une toux dans 6 cas et des crachats hémoptoiques dans 4 cas.

Le diagnostic d'un traumatisme laryngé fermé a été méconnu chez un seul patient qui a été admis initialement au service de réanimation pour polytraumatisme et intubé pendant 30 jours. Le diagnostic de traumatisme laryngé n'a été porté qu'ultérieurement, lors du bilan d'extubation de ce patient.

L'examen cervical, a trouvé un oedème cervical antérieur dans $50 \%$ des cas, une douleur à la mobilisation laryngée dans $24 \%$ des cas, une plaie soufflante dans 1 cas et un emphysème sous cutané dans 1 cas.

L'examen du larynx au nasofibroscope ou par le miroir laryngé a été pratiqué pour tous nos patients (100\%). La mobilité laryngée était normale dans $54 \%$ des cas. Un œdème laryngé a été trouvé dans $38 \%$ des cas et une luxation cricoaryténoïdienne dans $8 \%$ des cas.

La tomodensitométrie cervicale, réalisée chez 16 patients (54\%), a montré un œdème laryngé isolé dans 6 cas $(37 \%)$, une fracture du cartilage thyroïde dans 3 cas (27\%) (Fig. 1 et 2), du cartilage cricoïde dans 2 cas (Fig. $3)$, de l'os hyoïde dans 2 cas, et de la trachée dans 1 cas. Une luxation cricoaryténoïdienne a été notée dans 2 cas Au décours de ces examens, tous nos patients ont été classés selon la classification de Schaefer modifiée par Fuhrman (Tableau I). Plus que la moitié des malades (54\%) étaient classés en stade I et II. Les stades III et IV ont été respectivement trouvés dans 17 et $26 \%$ des cas.

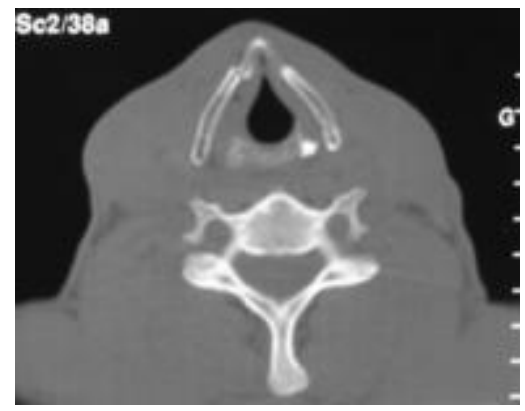

Fig 1 : TDM cervical en coupe axiale : fracture non déplacée du cartilage thyroïde

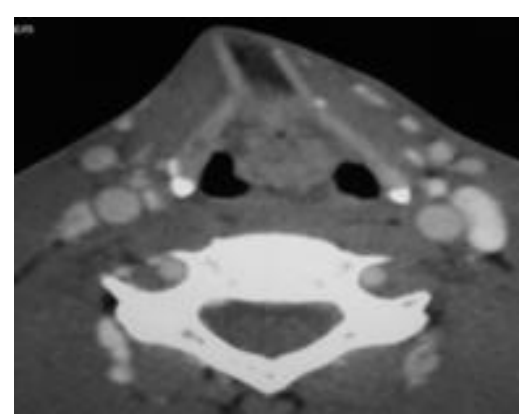

Fig 2 : TDM cervical en coupe axiale : fracture déplacée du cartilage thyroïde

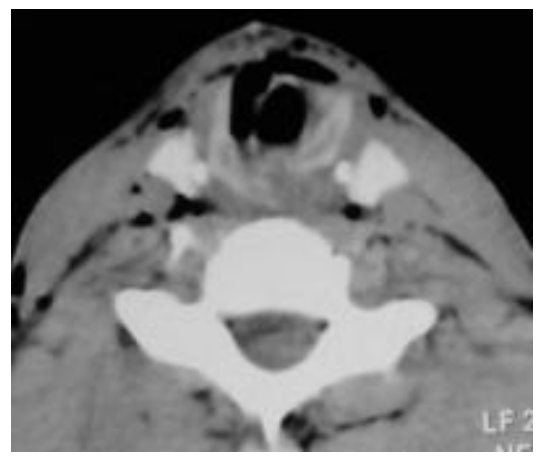

Fig 3 : TDM cervicale en coupe axiale : fracture complexe du cartilage cricoïde

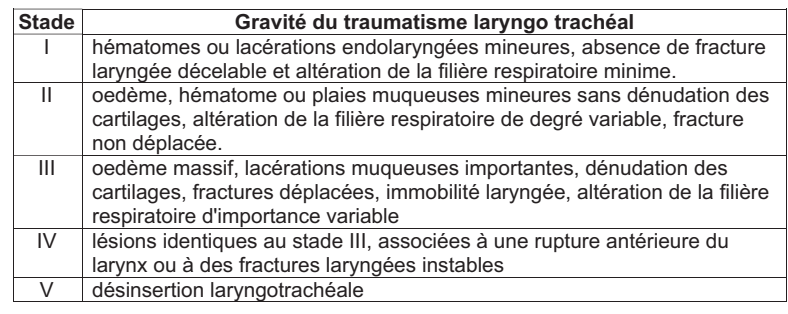

Tableau I : Classification de Schafer modifiée par Fuhmann

Un patients a été admis en extrême urgence pour traumatisme laryngé avec une dyspnée majeur et un emphysème sous cutané cervico-médistinal rapidement exten- 
sif. Ce patient a été rapidement acheminé au bloc opératoire. On a eu recours à une trachéotomie de sauvetage sous anesthésie locale dans un premier temps. Dés que son état respiratoire a été stabilisé, ce patient a eu une TDM cervico médiasinale montant de multiples fractures complexes des cartilages thyroïde et cricoïde associées à un oedème important des VAS, un emphysème sous cutané diffus et un pneumomédistin compressif et un pneumothorax gauche suffocant cadrant avec une désinsertion laryngotrachéale totale. Malgré nos tentatives de réparations de ces lésions, le patient a été décédé sur la table opératoire dans un tableau de choc cardiogénique. Afin d'assurer le contrôle des voies aériennes, on a eu recours à une trachéotomie sous anesthésie locale dans 11 cas $(37 \%)$ pour dyspnée grave. Ces patients avaient un traumatisme laryngotrachéale isolé et nous ont été ramenés à l'hôpital sans intubation préalable sur les lieus de l'accident.

La laryngoscopie directe sous anesthésie générale a été réalisée pour 25 patients. Elle a montré un œdème de la filière laryngée dans 9 cas, une luxation cricoaryténoïdienne dans $14 \%$ et une fracture des anneaux trachéaux dans $10 \%$ des cas.

L'exploration chirurgicale a été indiquée pour 16 patients. Elle a été réalisée par voie de cervicotomie à la 48éme heure. Les lésions endolaryngées rencontrées étaient essentiellement des fractures de l'os hyoïde dans 3 cas, du cartilage thyroïde dans 8 cas, du cartilage cricoïde dans 4 cas, de l'épiglotte dans 1 cas, des anneaux trachéaux dans 3 cas et une luxation cricoaryténoïdienne dans 4 cas.

Les lésions de l'axe viscéral du cou associées étaient à type de déchirure de la muqueuse pharyngée dans un cas de la muqueuse osophagienne dans 3 cas et un étirement du nerf récurrent dans un cas

Le traitement a été conservateur dans tous les cas. La remise en place des fracas cartilagineux a été effectuée dans 8 cas. Les luxations des cartilages cricoaryténoïdiens ont été réduites chez 2 patients.

Concernant la réparation des lésions des paries molles cervicales associées. Une suture de la muqueuse endolaryngée a été pratiquée dans 6 cas, une suture de la muqueuse œsophagienne dans 2 cas.

On a eu recours à un calibrage endolaryngé par un tube en $T$ de Montgomery dans 4 cas pour fracture instable du cartilage cricoïde. Ce tube a été enlevé au bout de quinze jours.

Une jéjunostomie d'alimentation a été pratiquée dans 1 cas pour fistule œsophagienne et elle a été gardée pendant 30 jours.

La décanulation a été faite dans un délai moyen de dix jours. Un seul patient a gardé une trachéotomie définitive pour sténose glotto-sous glottique sévère étendue avec immobilité laryngée.

Le recul moyen de nos patients est de 2 ans avec des extrêmes allant de 6 mois à 10 ans.

Concernant les complications tardives, trois de nos patients ont développé une sténose sous glottique grade II de Cotton. Ils ont eu une laryngoplastie d'agrandissement type Réthi avec de bons résultats dans deux cas.

Au cours du suivi, 22 patients ont gardé des séquelles phonatoires à type de dysphonie et une rééducation orthophonique leur a été indiquée. Néanmoins, 14 patients ont gardé une dysphonie légère. Une dyspnée d'effort a été notée chez 2 patients et des troubles de la déglutition chez 2 autres.

\section{DISCUSSION}

Les traumatismes externes du larynx sont rares. Chez l'adulte, leur incidence est estimé à 1/30 000, représentant moins de $1 \%$ des traumatismes aigus $(1,2)$. Ils sont responsables d'une mortalité dans l'immédiat dans $75 \%$. Ils peuvent se rencontrer à tout âge, mais plus volontiers chez l'adulte jeune en particulier de sexe masculin (1).

Ces traumatismes sont divisées en deux groupes: les traumatismes ouverts et les traumatismes fermés du larynx.

Les traumatismes ouverts représentent environ 15 à $20 \%$ des traumatismes laryngés et 0,2 à $8 \%$ des plaies du cou. Leurs étiologies sont dominées essentiellement par les tentatives d'autolyse et les agressions (3).

Les traumatismes fermés sont de loin les plus fréquents avec une incidence estimée de 80 à $85 \%$.Les accidents de la voie publique sont les plus pourvoyeurs de ces traumatismes $(57 \%)$ suivis des accidents sportifs et des tentatives d'autolyse par pendaison (4).

La symptomatologie varie suivant le type de traumatisme. Le diagnostic de traumatisme ouvert du larynx ne pose guère de problèmes, contrairement à celui d'un traumatisme fermé. En effet, dans $37 \%$ des cas, il s'intègre dans un contexte de polytraumatisme, et peut alors passer inaperçu, masqué par une intubation ou une trachéotomie réalisée en urgence, et ne sera découvert qu'au stade séquellaire (5).

Les signes fonctionnels sont inconstants et peuvent être diversement associés. La dyspnée, immédiate ou retardée, conditionne l'attitude à adopter. La dysphonie est quasi constante. Ailleurs on peut trouver une douleur à la déglutition, une toux, une dysphagie plus ou moins nette, associée à une hypersalivation traduisant une lésion oesophagienne. (6).

La palpation prudente peut trouver une perte des reliefs laryngés, une douleur exquise à la mobilisation d'un cartilage ou un emphysème sous-cutané traduisant la rupture de l'arbre trachéobronchique.

L'examen au nasofibroscope réalisable chez un patient non intubé, peu ou pas dyspnéique est l'examen de choix en urgence pour la plupart des auteurs. II permet d'apprécier la mobilité laryngée, l'état de la filière respiratoire, l'intégrité ou non de la muqueuse laryngée et la présence 
de cartilages dénudés (5).

Les radiographies cervicales simples de face et de profil, et en dehors des traumatismes nécessitant un geste d'urgence, sont réalisées systématiquement sans hyperextension, permettant surtout d'éliminer un traumatisme du rachis cervical (7).

La radiographie du thorax peut mettre en évidence un pneumomédiastin, un pneumothorax, un élargissement médiastinal ou un hydropneumothorax (8).

Actuellement, la plupart des auteurs s'accordent que la tomodensitométrie cervicale est l'examen de choix surtout dans les traumatismes fermés, ou dans les traumatismes peu importants ou d'importance moyenne. En effet, elle permet de visualiser parfaitement les cartilages et les lésions laryngées $(7,9)$. À distance, elle peut permettre de rattacher à un traumatisme méconnu ou négligé des lésions laryngées séquellaires (10).

Le contrôle des voies aériennes est une priorité pour les traumatismes laryngés. L'intubation immédiate comporte un risque important d'aggravation des lésions endolaryngées, de fausses routes et de décompensation d'une désinsertion laryngotrachéale et ne doit être réservée qu'aux traumatismes mineurs (absence de dyspnée, lésions mineures à l'examen au nasofibroscope, absence de fracture au scanner (11). La trachéotomie, réalisée sous anesthésie locale, est la technique de choix afin d'assurer une ventilation satisfaisante et la seule technique à utiliser en cas de dyspnée $(12,13)$.

L'attitude thérapeutique actuelle dans les traumatismes externes isolés du larynx, qu'ils soient ouverts ou fermés, est encore sujet de controverses. Plusieurs algorithmes ont été proposés par les auteurs afin de guider les indications d'un traitement chirurgical.

La majorité des données de la littérature s'accordent que les traumatismes mineurs relèvent d'un traitement médical et d'une hospitalisation pour surveillance de 24 heures minimum sous aérosolthérapie, corticothérapie et contrôle par oxymétrie de pouls (14).

Tout traumatisme plus important nécessite une exploration chirurgicale. Les buts de la chirurgie dans les traumatismes laryngotrachéaux sont de limiter le risque de sténose pour conserver la fonction respiratoire et de conserver la fonction phonatoire.

Le bilan endoscopique sous anesthésie générale réalisé après avoir éliminé de façon formelle une fracture du rachis cervical, constitue le premier temps avant tout geste chirurgical de réparation. II permet de mettre en évidence les lésions endolaryngées et d'explorer la sous glotte et la trachée. L'oesophagoscopie, est réalisée de façon systématique au décours du bilan endoscopique. L'heure de l'exploration chirurgicale est encore mal définie. Certains auteurs recommandent d'attendre 3 à 5 jours la régression de l'œdème. D'autres, ont montré que les meilleurs résultats respiratoires et vocaux sont obtenus lorsque le geste chirurgical est effectué dans les 24 premières heures.

Le traitement des lésions se fait de la profondeur vers la superficie en veillant à rétablir une architecture laryngée stable et fonctionnelle $(14,15)$.

Le principe du calibrage endolaryngé ou endotrachéal répond au souci de conservation de la perméabilité de la filière respiratoire. Toutefois, son indication est discutée et il est utilisé avec parcimonie. En effet, il nécessite le maintien d'une trachéotomie qui favorise les surinfections locales, et peut entretenir des lésions muqueuses notamment lors des mouvements laryngés induits par la déglutition.

En pratique, si la couverture muqueuse est bonne et la réparation du squelette laryngé stable, l'utilisation d'un calibrage est inutile, voire néfaste si le larynx est mobile. Les procédés de calibrage endoluminal sont réservés aux fractures instables, pour assurer une contention interne des fragments cartilagineux ; en cas de délabrements muqueux importants pour limiter le risque de synéchies ; en cas de désinsertion laryngotrachéale car il existe souvent un délabrement cricoïdien associé ; en cas de fracture de la trachée cervicale, par une trachéotomie réalisée au-dessus de la lésion. Le maintien ne doit pas excéder 15 jours, même si certains auteurs les conservent 3 à 6 semaines.

Les lésions associées seront traitées dés que possibles en même temps opératoire.

Les plaies pharyngées et oesophagiennes sont suturées avec ou sans lambeau musculaire d'interposition. L'alimentation est assurée par mise en place d'une sonde nasogastrique.

Les plaies des nerfs laryngés inférieurs sont suturées aidée par des lunettes-loupes ou un microscope opératoire doit être tentée, même si le résultat est constamment décevant. II a été décrit une réimplantation directe dans le muscle cricoaryténoïdien postérieur.

Les plaies vasculaires sont associées aux plaies du cou dans un tiers des cas. Elles sont à rechercher de principe. Elles sont le plus souvent rencontrées dans la zone cervicale I qui va du rebord de la clavicule au cartilage cricoïde.

Les lésions de la face seront réparées après stabilisation et réparation des lésions aérodigestives (16).

En post opératoire, l'antibioprophylaxie est systématiquement prescrite dans les traumatismes stades III, IV et V pendant 2 à 10 jours (17). Les céphalosporines sont les plus indiquées.

L'aérosolthérapie est indispensable pour humidifier l'air inspiré, ainsi que des soins de trachéotomie pluriquotidiens (17).

Les IPP seraient intéressants afin de lutter contre un éventuel reflux gastro oesophagien qui risque de retarder la bonne cicatrisation des lésions (18).

La surveillance clinique ultérieure fait appel à l'examen en laryngoscopie indirecte, et surtout à l'examen au nasofi- 
broscope, afin de surveiller l'évolution et de dépister la survenue d'une éventuelle sténose. En effet les traumatismes larygotrachéales peuvent se compliquer de sténoses laryngées et de dysphonie dans $40 \%$ des cas (19). La rééducation orthophonique a pour but d'optimiser les résultats fonctionnels et ne doit pas être négligée (20).

\section{CONCLUSION}

Les traumatismes du larynx ont un taux de mortalité et de morbidité élevé. Au cours des dernières décennies, une stratégie diagnostique et thérapeutique bien codifiée, a été définie, ce qui devrait permettre une amélioration de la prise en charge de ces traumatismes. Seuls un diagnostic et un traitement précoces et adaptés peuvent permettre la préservation ou la restitution des fonctions respiratoires et phonatoires du larynx et éviter l'apparition de sténoses.

\section{REFERENCES}

1) Jewett BS, Schockley VW, Rutletge R External laryngeal trauma analysis of 392 patients. Arch Otolaryngol Head Neck Surg $1999 ; 125$ : $877-880$

2) Ganapathy Dhanasekar, Maziar Sadri, Sharat Mohan, Kate Young, Jacob Leiberman. Blunt laryngeal trauma resulting in arytenoids dislocation and dysphonia. Auris Nasus Larynx 2006 ; 33 : 75-8

3) Grewal H, Prakashchandra MR, Mukerii S, Ivatury RR Management of penetrating laryngeal injuries. Head Neck $1995 ; 17: 494-502$

4) Delaere $P$, Feenstra $L$ Management of acute laryngeal trauma. Acta Otorhinolaryngol Belg $1995 ; 49$ : $347-9$

5) Johannes J. Kuttenberger1, Nicolas Hardt1, Christoph Schlegel Diagnosis and initial management of laryngotracheal injuries associated with facial fractures. J Cranio-Maxillofacial Surgery 2004; 32 : 80-4

6) Ford HR, Gardner MJ, Lynch JM Laryngotracheal disruption from blunt pediatric neck injuries: impact of early recognition and intervention on outcomes. $J$ Pediatr Surg 1995; 30: 331-5

7) Mariano S, Stefania R, Antonio P, Amelia S, Michele S, Antonio R. Acute tracheobronchial injuries: Impact of imaging on diagnosis and management implications. European Journal of Radiology 2006 ; 59: 336-43

8) Schoem SR, Choi SS, Zalzal GH Pneumomediastinum and pneumothorax from blunt cervical trauma in children. Laryngoscope $1997 ; 107: 351-6$

9) Athanasios P, Zinovia A, Konstantinos S, Konstantinos Ch. Diagnostic findings in laryngeal fracture. Injury Extra $2006 ; 37,425-7$

10) Yoshihiro M, Mitsugi S, Goro M, , Noriyuki S. Usefulness of the 3Dimensionally Reconstructed Computed Tomography Imaging forDiagnosis of the Site of Tracheal Injury. World J. Surg $2005 ; 29,102-5$

11) Bent JP, Silver JR, Porubsky ES Acute laryngeal trauma: a review of 77 patients. Otolaryngol Head Neck Surg $1993 ; 109: 441-9$
12) Lacau St Guily J, Périé $S$ Conduite à tenir face à un traumatisme externe et une plaie du cou. In: Les urgences en ORL. Paris: Société française d'oto-rhinolaryngologie et de chirurgie de la face et du cou: 2002; 353-63.

13) Murakawa T, Nakajima J, Motomura N, Murakami A, Takamoto S Successful allotransplantation of cryopreserved tracheal grafts with preservation of the pars membranacea in non human primates. J. Thorac. Cardiovasc. Surg. 2002 ; 123 : $153-60$

14) Todd Preston, MD, Fred G. Fedok, MD. Blunt and penetrating trauma to the larynx and upper airway. Operative Techniques in Otolaryngology $2007 ; 18$ : 140-3

15) Goldenberg D, Golz A, Flax-Goldenberg R, Joachims HZ Severe laryngeal injury caused by blunt trauma to the neck: a case report. J Laryngol Otol 1997; 111: 1174-6

16) Amin M, Dill-Russel P, Manisali M, Lee R, Sinton I Facial fractures and sub mental tracheal intubation. Anaesthesia $2003 ; 58: 496-7$

17) Schoem SR, Choi SS, Zalzal GH Pneumomediastinum and pneumothorax from blunt cervical trauma in children. Laryngoscope $1997 ; 107: 351-6$

18) David S. Verschueren R. Bryan B, Shahrokh C. Management of LaryngoTracheal

Injuries Associated With Craniomaxillofacial Trauma. J Oral Maxillofac Surg $2006 ; 64$ : 203-14

19) llias K, Dimitrios G, Nikolaos K, Michael K, Maria R, Vasilis D. The infuence of laryngopharyngeal refux in the healing of laryngeal trauma. Eur Arch Otorhinolaryngol. 2009;266:253-9

20) Brosch S, Johanssen HS Clinical course of acute laryngeal trauma and associated effects on phonation. J Laryngol Otol 1999 ; 113 : 58-61 